\title{
Large Bragg Reflection from One-Dimensional Chains of Trapped Atoms Near a Nanoscale Waveguide
}

\author{
Neil V. Corzo, ${ }^{1}$ Baptiste Gouraud, ${ }^{1}$ Aveek Chandra, ${ }^{1}$ Akihisa Goban, ${ }^{2, *}$ Alexandra S. Sheremet, ${ }^{3,4}$ \\ Dmitriy V. Kupriyanov, ${ }^{5}$ and Julien Laurat ${ }^{1, \dagger}$ \\ ${ }^{1}$ Laboratoire Kastler Brossel, UPMC-Sorbonne Universités, CNRS, ENS-PSL Research University, \\ Collège de France, 4 Place Jussieu, 75005 Paris, France \\ ${ }^{2}$ Norman Bridge Laboratory of Physics 12-33, California Institute of Technology, Pasadena, California 91125, USA \\ ${ }^{3}$ Information Technologies, Mechanics and Optics University, 199034 St. Petersburg, Russia \\ ${ }^{4}$ Russian Quantum Center, 143025 Skolkovo, Moscow Region, Russia \\ ${ }^{5}$ Department of Theoretical Physics, St. Petersburg State Polytechnic University, 195251 St. Petersburg, Russia
}

(Received 7 April 2016; published 23 September 2016)

\begin{abstract}
We report experimental observations of a large Bragg reflection from arrays of cold atoms trapped near a one-dimensional nanoscale waveguide. By using an optical lattice in the evanescent field surrounding a nanofiber with a period nearly commensurate with the resonant wavelength, we observe a reflectance of up to $75 \%$ for the guided mode. Each atom behaves as a partially reflecting mirror and an ordered chain of about 2000 atoms is sufficient to realize an efficient Bragg mirror. Measurements of the reflection spectra as a function of the lattice period and the probe polarization are reported. The latter shows the effect of the chiral character of nanoscale waveguides on this reflection. The ability to control photon transport in 1D waveguides coupled to spin systems would enable novel quantum network capabilities and the study of many-body effects emerging from long-range interactions.
\end{abstract}

DOI: 10.1103/PhysRevLett.117.133603

In recent years, the coupling of one-dimensional bosonic waveguides and atoms, either real or artificial, has raised a large interest [1-3]. Beyond the remarkable ability to couple a single emitter to a guided mode [3], the 1D reservoir would also enable the exploration and eventual engineering of photon-mediated long-range interactions between multiple qubits, a challenging prospect in free-space geometries. This emerging field of waveguide quantum electrodynamics promises unique applications to quantum networks, quantum nonlinear optics, and quantum simulation [4-6].

In this context, progress has been reported on various fronts. In the microwave regime, the coupling of superconducting qubits to a one-dimensional transmission line provides a versatile platform to study such photon-mediated interactions [2]. At optical frequencies, recent experimental advances include the development of 1D nanoscale dielectric waveguides coupled to cold atoms trapped in the vicinity [7-9]. In these experiments, tight transverse confinement of the electric field achieves an effective mode area comparable to the atomic cross section and thereby a strong atom-photon interaction in a single-pass configuration [10].

Coupling of atom arrays to 1D waveguides could lead to a variety of remarkable cooperative phenomena. This coupling can strongly modify the photon transport properties [11-13], resulting for instance in sub- and superradiant decays as recently observed for two coupled atoms [14]. It can also lead to photonic band gaps and provide atomic Bragg mirrors, with envisioned applications to integrated cavity QED [15-17]. This setting is as well at the basis of a recently proposed deterministic state engineering protocol [18] and constitutes the building block of chiral spin networks in which the emission into the left- and rightpropagating modes is asymmetric [19]. Moreover, strong optomechanical couplings resulting from photon-mediated forces can give rise to rich spatial atomic configurations, including self-organization [20,21].

Optical nanofibers offer a promising platform for exploring these effects. Their subwavelength diameter results in a large evanescent field that can be used for both trapping and interacting with atoms [22]. Seminal works achieved the trapping of cold atoms near an optical nanofiber $[7,8]$ and recently enabled the demonstrations of all-fibered optical memories $[23,24]$. These works were realized with disordered atoms or incommensurate arrays, and relied on the optical depth of the medium. An important capability that has not been demonstrated heretofore is the realization of cooperative effects emerging from the spatial order of the atoms, when the lattice is commensurate or close to commensurate with the resonant wavelength.

In this Letter, we report a large Bragg reflection from atom arrays near a one-dimensional waveguide in a situation where each trapping site is occupied by at most one atom. Due to the tight transverse confinement of the guided light, a few thousand atoms trapped in the evanescent field of a nanofiber are sufficient to strongly reflect the incoming light. By using a near-resonant dipole trap, the maximum Bragg reflection is obtained for a slightly detuned probe. We provide detailed characterizations of the reflection 
spectra and finally show the effect of the waveguide chirality arising from the complex polarization pattern of tightly focused light.

The long-range order of trapped atoms can indeed dramatically change the scattering properties. In incommensurate arrays, the interference between forward- and backward-scattered light leads to absorption and a vanishing reflection. In contrast, in commensurate arrays, this interference can result in strong reflection close to resonance [25]. This effect, well-known as Bragg reflection, has been largely studied in crystals as well as in multilayer dielectric structures. It has also been observed with ordered cold atoms in free space, either with three-dimensional $[26,27]$ or onedimensional optical lattices [28,29]. A reflectance as high as $80 \%$ was demonstrated in [29]. This observation required around $10^{7}$ atoms distributed over 7700 layers to reach the regime of multiple reflections. In contrast, in this work, we demonstrate that 2000 atoms are sufficient to achieve large reflectance in a waveguide-mediated scenario.

To introduce the scattering properties and associated photon transport, we first consider a typical configuration as depicted in Fig. 1(a). $N$ atoms are trapped in the vicinity of a waveguide, with a lattice constant $d$ close to $\lambda_{0} / 2$, where $\lambda_{0}$ correponds to the wavelength of the atomic transition. Because of the complex polarization structure of tightly focused light [30], which includes a significant longitudinal component, the scattering in the guided mode can be asymmetric [31]. Each atom exhibits a radiative decay rate $\Gamma_{1 \mathrm{D}}^{\text {forw }}$ and $\Gamma_{1 \mathrm{D}}^{\text {back }}$ into the right- and left-propagating mode, respectively, and $\Gamma^{\prime} \simeq \Gamma_{0}$ into all the other modes. $\Gamma_{0}$ is the radiative decay rate in free space. For a guided probe field quasilinearly polarized along the $y$ direction, the two decay rates are equal, $\Gamma_{1 \mathrm{D}}^{\text {back }}=\Gamma_{1 \mathrm{D}}^{\text {forw }}=\Gamma_{1 \mathrm{D}} / 2$. For an orientation along the $x$ direction, i.e., pointing towards the atoms, the couplings to the waveguide become strongly asymmetric. It has also been shown that when the ground state Zeeman levels are equally populated, the two $x$ and $y$ polarizations are not coupled to each other by the linear coherent scattering [32]. For nanofiber-trapped atoms, typically around $200 \mathrm{~nm}$ from the surface, the ratio $P=\Gamma_{1 \mathrm{D}} / \Gamma_{0}$ amounts to a few $10^{-2}$. In the case of asymmetric coupling, the forward decay rate is increased by sixfold while the backward decay rate is suppressed by about one order of magnitude.

The single-atom amplitude reflection $r$ can be calculated from the couplings to the waveguide and the probe detuning $\delta$ to resonance. In the symmetric decay case, for instance, the reflection is given by $r \simeq-\Gamma_{1 \mathrm{D}} /\left(\Gamma_{0}-2 i \delta\right)$ [1]. At resonance, one atom thus reflects $P^{2} \simeq 10^{-4}$ in intensity. The transfer matrix formalism [25] enables then to calculate the single-photon propagation through the ensemble (see Supplemental Material (SM) [33]).

Figures 1(c) and 1(d) provide theoretical reflection spectra for different small detunings $\Delta \lambda$ of the trap wavelength to atomic resonance and for the two orthogonal polarizations. For atoms separated exactly by $\lambda_{0} / 2$, the reflection spectrum is a broadened Lorentzian in the (a)
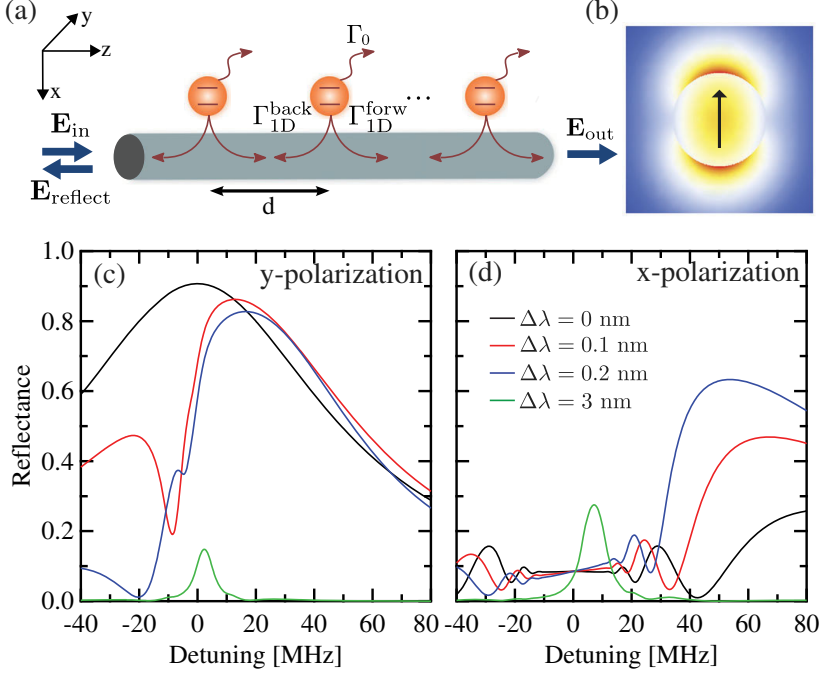

FIG. 1. Bragg reflection from atoms coupled to a onedimensional waveguide. (a) $N$ atoms are trapped near of a waveguide and exhibit radiative decay rates $\Gamma_{1 \mathrm{D}}^{\text {forw,back }}$ into the right- and left-propagating modes, and $\Gamma^{\prime} \simeq \Gamma_{0}$ into all the other modes. (b) Electric field distribution in the transverse plane of a nanofiber for a guided probe with a quasilinear polarization (indicated by the arrow). (c) Theoretical reflection spectra for a probe quasilinearly polarized along the $y$ direction (symmetric decay rates) and (d) along the $x$ direction (asymmetric decay rates). The spectra are given for different distances between the atoms, with values close to the commensurate case. $\Delta \lambda$ stands for the trap detuning to resonance, with $d=\lambda_{0} / 2+\Delta \lambda / 2$. $\left(N=2000, \Gamma_{1 \mathrm{D}} / \Gamma_{0}=0.01, \Gamma_{1 \mathrm{D}}^{\text {forw }}=2.8 \Gamma_{1 \mathrm{D}}, \Gamma_{1 \mathrm{D}}^{\text {forw }} / \Gamma_{1 \mathrm{D}}^{\text {back }}=12\right)$. Theoretical values of the couplings are taken from [31].

symmetric coupling case while the reflectance is strongly suppressed in the chiral one. Indeed, the amount of chirality and number of atoms result in a finite bandwidth around resonance where reflection is suppressed, as detailed in [32]. For close-to-commensurate traps, as studied here, the Bragg condition is fulfilled out of resonance. This leads to a maximum reflectance shifted to the blue [29] but also results in an increased reflectance for the chiral case. Large reflectance values can then be obtained for both polarizations as the single-atom reflection coefficients are similar in our configuration.

Our experimental setup is illustrated in Fig. 2. Arrays of trapped atoms are prepared in the evanescent field of a 400-nm diameter nanofiber suspended inside an ultrahigh vacuum chamber. The nanofiber is produced from a singlemode fiber (OZ Optics SMF-780-5/125) by the standard heating-pulling technique. The polarization of the different guided beams can be aligned by measuring the polarization properties of the light scattered from imperfections at the fiber surface $[8,36]$.

Our two-color dipole trap is based on the combination of laser beams all guided by the nanofiber. By using the appropriate combination of attractive red-detuned light and repulsive blue-detuned light, the atoms are trapped in the potential minima located at a subwavelength distance from 


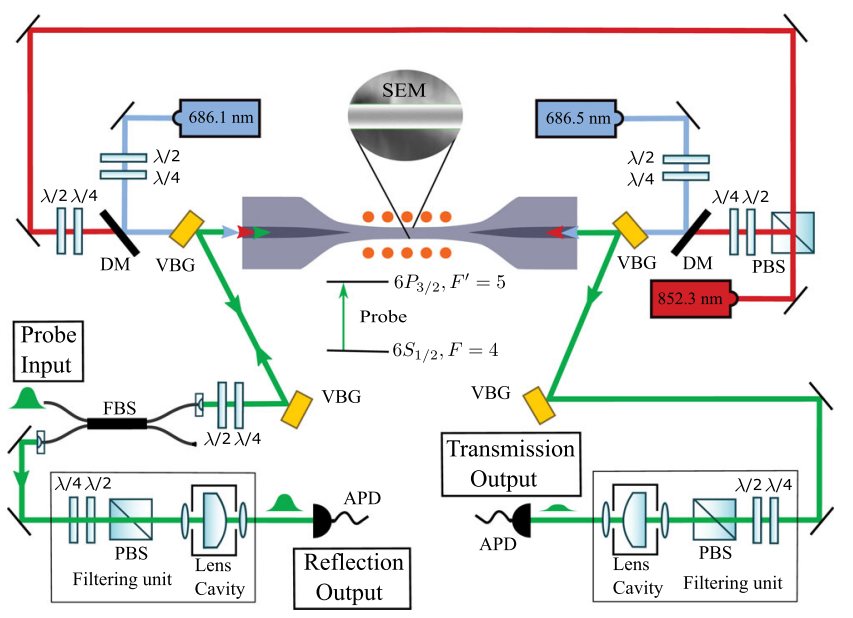

FIG. 2. Experimental setup. Arrays of cesium atoms are trapped in the evanescent field of a nanofiber. The lattice is realized by a pair of close-to-resonance red-detuned counterpropagating beams. An additional pair of blue-detuned beams with slightly different wavelengths gives a repulsive contribution. The atoms are prepared in the $|g\rangle=\left\{6 S_{1 / 2}, F=4\right\}$ ground state and the probe addresses the transition $|g\rangle \rightarrow|e\rangle=\left\{6 P_{3 / 2}, F^{\prime}=5\right\}$. Around 2000 atoms in total are trapped along two parallel chains. Reflection and transmission spectra are measured with avalanche photodiodes after polarization and frequency filterings. DM stands for dichroic mirror, FBS for fiber beam splitter, and VBG for volume Bragg grating.

the waveguide $[7,8,37]$. The longitudinal periodic structure is given by the standing wave formed by the two counterpropagating red-detuned beams, with $\Delta \lambda$ the detuning from resonance. We perform our experiment at two different values of $\Delta \lambda, 0.12 \mathrm{~nm}$ and $0.2 \mathrm{~nm}$, with a total power equal to $2 \times 1 \mu \mathrm{W}$ and $2 \times 1.9 \mu \mathrm{W}$, respectively. A pair of counterpropagating beams $(2 \times 4 \mathrm{~mW})$ at $686.1 \mathrm{~nm}$ and $686.5 \mathrm{~nm}$ are chosen as blue-detuned beams and contribute to the trap in a compensated manner [8]. The polarization of the beams is oriented along the transverse $x$ direction. The resulting intensity pattern leads to trapping sites aligned along two lines parallel to the fiber. Because of the loading dynamics, each site hosts one atom at most [38]. Simulations of the trapping potential [33] provide for $\Delta \lambda=$ $0.12 \mathrm{~nm}(0.2 \mathrm{~nm})$ a trap depth at minimum equal to $-0.15 \mathrm{mK}(-0.1 \mathrm{mK})$ and an axial trap frequency $\nu_{z} / 2 \pi=$ $258 \mathrm{kHz}(215 \mathrm{kHz})$. For $\Delta \lambda=0.2 \mathrm{~nm}$, we measure a shift of the transition equal to $3 \mathrm{MHz}$ relative to free space and an inhomogeneous broadening limited to $\sigma=0.6 \Gamma_{0}$.

To load the dipole trap, which is continuously on, we overlap a cigar-shaped magneto-optical trap (MOT) along the fiber axis. The atomic cloud is then further cooled and the lattice is loaded during an optical molasses phase: the MOT coils are switched off, and during a $10 \mathrm{~ms}$ interval, the MOT cooling-beam detuning is increased from $-2 \Gamma_{0}$ to $-16 \Gamma_{0}$, while the powers of both the cooling and repumping beams are decreased to zero. The atoms are then optically pumped into the $|g\rangle=\left\{6 S_{1 / 2}, F=4\right\}$ hyperfine ground state for $400 \mu \mathrm{s}$. By a saturation measurement, we estimate the number of trapped atoms to be $N=2000 \pm 200$. Residual magnetic fields are canceled with bias coils and characterized via Zeeman sublevel microwave spectroscopy. The fields are compensated down to the $20 \mathrm{mG}$ level.

To measure the reflection and transmission spectra, a probe pulse first passes through a fibered beam splitter that we use to separate the reflected pulse from the input probe. The probe is then combined with the pair of trapping beams, via a volume Bragg grating (VBG), and sent into the nanofiber. The weak probe pulse arriving on the atoms has a mean photon number of $2 \pm 0.05$. The transmitted pulse is filtered by another VBG and an additional filtering system, and finally detected by an avalanche photodiode. The filtering system combines a polarization beam splitter and a commercial lens-based cavity [39]. The cavity transmission is around $75 \%$, with a bandwidth of $80 \mathrm{MHz}$ and a rejection around $40 \mathrm{~dB}$ for the trapping beams. These cascaded stages are required to filter out efficiently the dipole beams and reach the single-photon level. The reflected pulse is directed to a similar filtering and detection stage.

The reflectance values are obtained by comparing the reflected pulse when atoms are trapped to the transmitted pulse without atoms, and by correcting for the different losses in the system. These losses, which are detuning dependent due to the filtering cavities, are calibrated before and after each measurement. The transmittance values are obtained by comparing the transmitted pulse with and without trapped atoms. Error bars in the data include the calibration uncertainties.

We first characterize the reflection and transmission spectra for a probe quasilinearly polarized along the $y$ direction. In this configuration, the radiative decay rates are expected to be symmetric. The measurements are given in Fig. 3 for the two different detunings of the dipole trap. For $\Delta \lambda=0.12 \mathrm{~nm}$,
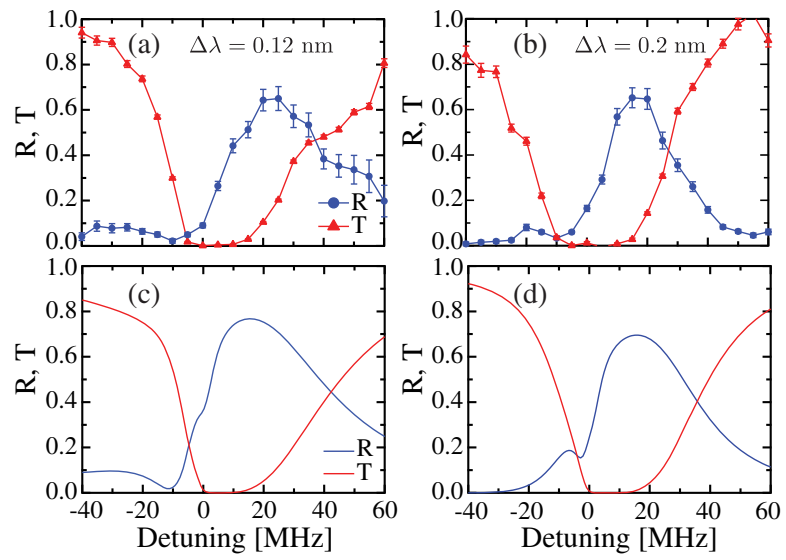

FIG. 3. Reflection and transmission spectra for a probe quasilinearly polarized along the $y$ direction. (a) and (b) Experimental results for $\Delta \lambda=0.12 \mathrm{~nm}$ and $\Delta \lambda=0.2 \mathrm{~nm}$. (c) and (d) Simulated spectra for $N=2000$ atoms, $\Gamma_{1 \mathrm{D}} / \Gamma_{0}=0.007$, and a filling factor of the lattice sites $f=0.3$. The coupling value and filling factor have been adjusted to fit the measured spectra reported in (a). 


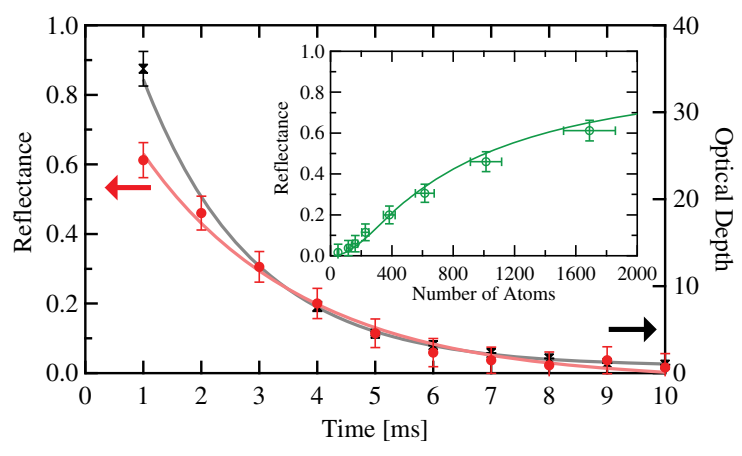

FIG. 4. Decay of the reflectance with trapping time and atom number. The largest measured reflectance (red) and the optical depth of the medium at resonance (black) are given as a function of the trapping time. The solid lines are exponential decay fits. The inset provides the reflectance as a function of the remaining number of trapped atoms. The green line is given by a simple model taking into account an initial filling factor $f=0.3$ and subsequent random loss $\left(\Delta \lambda=0.2 \mathrm{~nm}, \Gamma_{1 \mathrm{D}} / \Gamma_{0}=0.007\right)$.

as shown in Fig. 3(a), we observe a broad reflection spectrum, with a maximum reflectance of $(0.65 \pm 0.05)$ for a detuning of $25 \mathrm{MHz}$. For $\Delta \lambda=0.2 \mathrm{~nm}$, the reflection peak is narrower, and the maximum reflectance $(0.65 \pm 0.04)$ is shifted to a lower frequency [Fig. 3(b)].

The spectra resulting from the two trapping distances are well explained by our simple theoretical model, as presented in Figs. 3(c) and 3(d). In this model, we take into account the disorder in the distribution of the atoms along the two parallel arrays due to a limited filling factor $f$ of the individual trapping sites [33]. The coupling to the waveguide and the filling factor have been adjusted to provide the best fit to the spectra of Fig. 3(a). A value $f=0.3$ was obtained. Larger values lead to a pronounced dip on the reflection spectrum while smaller values result in a strongly reduced reflectance and a narrowing of the spectra width. The simulations take also into account the shift induced by the dipole trapping. Inhomogeneous broadening is not included as the limited broadening $\sigma$ leads to a negligible modification of the spectra [33].

Apart from a finite filling factor, a second contribution to disorder can arise from the thermal distribution of the atoms in the potential wells. The good agreement between the achieved reflectances and the predicted ones supports a tight axial localization, as expected with our microscopic traps. With a temperature $T=20 \mu \mathrm{K}$ estimated by a time-of-flight measurement and the predicted axial trap frequency of $\nu_{z} / 2 \pi=258 \mathrm{kHz}$, the spread is given in the harmonic approximation by $\sigma_{z}=\sqrt{\left(k_{B} T\right) /\left(m_{C s} \nu_{z}^{2}\right)} \sim 22 \mathrm{~nm}$, i.e., $\sim \lambda / 40$. This value leads to a Debye-Waller factor $f_{\mathrm{DW}}=$ $e^{-4 k^{2} \sigma_{z}^{2}}$ close to 0.9 [40]. As shown in the SM [33], this disorder has a very limited effect in our configuration where Bragg reflection is observed out of resonance [41].

The measurements described above were performed $1 \mathrm{~ms}$ after loading the trap. We now investigate the reflection for longer trapping time and compare its decay

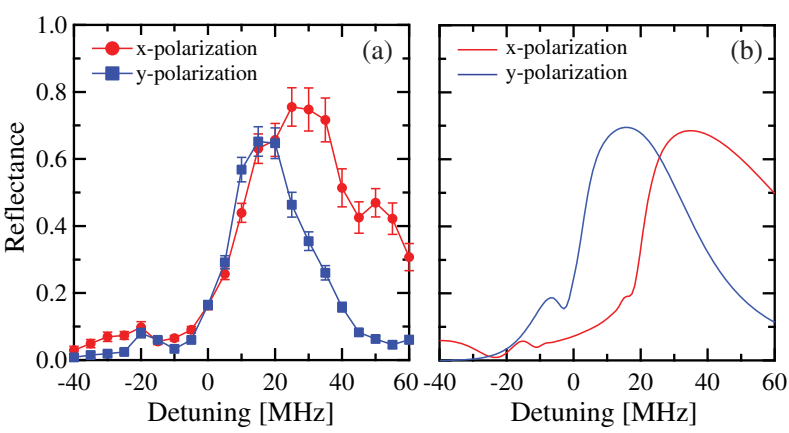

FIG. 5. Effect of the chiral character of the waveguide on Bragg reflection. (a) Measured reflection spectra for $x$ - and $y$-quasilinear polarization, with $\Delta \lambda=0.2 \mathrm{~nm}$. (b) Theoretical simulations $\left(N=2000, \quad \Gamma_{1 \mathrm{D}} / \Gamma_{0}=0.007, \quad \Gamma_{1 \mathrm{D}}^{\mathrm{forw}}=2.8 \Gamma_{1 \mathrm{D}}, \quad \Gamma_{1 \mathrm{D}}^{\text {forw }} / \Gamma_{1 \mathrm{D}}^{\text {back }}=12\right.$, $f=0.3$ ).

with the trap lifetime. Figure 4 shows the maximal reflectance and optical depth of the medium as a function of the hold time. Using exponential fits, we obtain a decay time of $2.7 \mathrm{~ms}$ and $1.9 \mathrm{~ms}$, respectively. From the measured optical depth, we then estimate the number of remaining trapped atoms at each time. The inset in Fig. 4 finally provides the maximal reflectance as a function of this inferred atom number. The green line takes into account a random loss of the atoms from the initial arrays with a total atom number of 2000 and a filling factor $f=0.3$ [33]. This model agrees well with the data.

Finally, we investigate the effect of the chiral character of the waveguide. The previous measurements were realized with the $y$ polarization, which leads to symmetric decay rates for the backward and forward scattering. We now consider the asymmetric case. This situation can be obtained with a probe quasilinearly polarized along the $x$ direction. The measured reflection spectra for both probe polarizations are compared in Fig. 5(a). The trap detuning is fixed at $\Delta \lambda=0.2 \mathrm{~nm}$. As it can be seen, the spectrum is significantly shifted and broadened in the asymmetric case. These features are compelling signatures of the chiral character of the waveguide on the reflection, as confirmed by the associated simulations in Fig. 5(b). The maximal observed reflectance of $(0.75 \pm 0.06)$ is obtained in this asymmetric case, at a probe detuning of $25 \mathrm{MHz}$.

In conclusion, we have realized an efficient Bragg atomic mirror based on a nanoscale one-dimensional waveguide coupled to about 2000 atoms. The effect of the chiral character of the waveguide on the reflection features has also been observed. Beyond their fundamental significance, these observations demonstrate key ingredients for the exploration of a variety of emerging and potentially rich protocols based on 1D reservoirs coupled to atoms. To enable an exact commensurate array of trapped atoms, and therefore an enhanced reflection closer to resonance, a bichromatic optical superlattice, whose trap periodicity is given by the tunable beat frequency of the trapping lasers $[42,43]$, can be developed. Such a trapping scheme, which has been demonstrated 
in free space, would need to be adapted to the evanescent field configuration around a nanofiber. This superlattice could also include a double primitive cell enabling a richer photonics spectrum [44]. An additional classical driving field in a threelevel atomic configuration would finally provide a dynamical control of the transport properties [45].

This work is supported by the European Research Council (Starting Grant HybridNet), the Emergence program from Ville de Paris, and the IFRAF DIM NanoK from Région Ile-de-France. The authors acknowledge interesting discussions within the CAPES-COFECUB Project No. Ph 740-12 and with H. J. Kimble when J. L. was in a visiting professor position at Caltech. The authors also thank A. Nicolas, D. Maxein, and O. Morin for their contributions in the early stage of the experiment, and A. C. McClung for constructive comments on the Letter. A. S. and D. V. K. acknowledge RFBR (Grant No. 15-02-01060). N. V. C. is supported by the EU (Marie Curie Fellowship). J. L. is a member of the Institut Universitaire de France.

Note added.-Recently, a related experiment by H.L. Sørensen and coworkers was presented in [46].

*Present address: JILA, University of Colorado, 440 UCB, Boulder, Colorado 80309, USA. julien.laurat@upmc.fr

[1] D. E. Chang, A. S. Sørensen, E. A. Demler, and M. D. Lukin, A single-photon transistor using nanoscale surface plasmons, Nat. Phys. 3, 807 (2007).

[2] A. L. van Loo, A. Fedorov, K. Lalumière, B. C. Sanders, A. Blais, and A. Wallraff, Photon-mediated interactions between distant artificial atoms, Science 342, 1494 (2013).

[3] P. Lodahl, S. Mahmoodian, and S. Stobbe, Interfacing single photons and single quantum dots with photonic nanostructures, Rev. Mod. Phys. 87, 347 (2015).

[4] H. J. Kimble, The quantum internet, Nature (London) 453 , 1023 (2008).

[5] D. E. Chang, V. Vuletić, and M. D. Lukin, Quantum nonlinear optics - photon by photon, Nat. Photonics 8, 685 (2014).

[6] A. González-Tuleda, C.-L. Hung, D. E. Chang, J. I. Cirac, and H. J. Kimble, Subwavelength vacuum lattices and atomatom interactions in two-dimensional photonic crystals, Nat. Photonics 9, 320 (2015).

[7] E. Vetsch, D. Reitz, G. Sagué, R. Schmidt, S. T. Dawkins, and A. Rauschenbeutel, Optical Interface Created by LaserCooled Atoms Trapped in the Evanescent Field Surrounding an Optical Nanofiber, Phys. Rev. Lett. 104, 203603 (2010).

[8] A. Goban, K. S. Choi, D. J. Alton, D. Ding, C. Lacroûte, M. Pototschnig, T. Thiele, N. P. Stern, and H. J. Kimble, Demonstration of a State-Insensitive, Compensated Nanofiber Trap, Phys. Rev. Lett. 109, 033603 (2012).

[9] A. Goban et al., Atom-light interactions in photonic crystals, Nat. Commun. 5, 3808 (2014).

[10] P. Domokos, P. Horak, and H. Ritsch, Quantum description of light-pulse scattering on a single atom in waveguides, Phys. Rev. A 65, 033832 (2002).
[11] J. T. Shen and S. Fan, Coherent photon transport from spontaneous emission in one-dimensional waveguides, Opt. Lett. 30, 2001 (2005).

[12] D. Dzsotjan, A. S. Sorensen, and M. Fleischhauer, Quantum emitters coupled to surface plasmons of a nanowire: a Green's function approach, Phys. Rev. B 82, 075427 (2010).

[13] F. Le Kien and A. Rauschenbeutel, Propagation of nanofiber-guided light through an array of atoms, Phys. Rev. A 90, 063816 (2014).

[14] A. Goban, C.-L. Hung, J. D. Hood, S.-P. Yu, J. A. Muniz, O. Painter, and H. J. Kimble, Superradiance for Atoms Trapped along a Photonic Crystal Waveguide, Phys. Rev. Lett. 115, 063601 (2015).

[15] D. E. Chang, L. Jiang, A. V. Gorshkov, and H. J. Kimble, Cavity QED with atomic mirrors, New J. Phys. 14, 063003 (2012).

[16] Y. Chang, Z. R. Gong, and C. P. Sun, Multiatomic mirror for perfect reflection of single-photons in a wide band of frequency, Phys. Rev. A 83, 013825 (2011).

[17] P.-O. Guimond, A. Roulet, H. Nguyen Le, and V. Scarani, Rabi oscillation in a quantum cavity: Markovian and nonMarkovian dynamics, Phys. Rev. A 93, 023808 (2016).

[18] A. González-Tudela, V. Paulisch, D. E. Chang, H. J. Kimble, and J. I. Cirac, Deterministic Generation of Arbitrary Photonic States Assisted by Dissipation, Phys. Rev. Lett. 115, 163603 (2015).

[19] H. Pichler, T. Ramos, A. J. Daley, and P. Zoller, Quantum optics of chiral spin networks, Phys. Rev. A 91, 042116 (2015).

[20] J. K. Asbóth, H. Ritsch, and P. Domokos, Optomechanical coupling in one-dimensional optical lattice, Phys. Rev. A 77, 063424 (2008).

[21] D. E. Chang, J. I. Cirac, and H. J. Kimble, Self-Organization of Atoms along a Nanophotonic Waveguide, Phys. Rev. Lett. 110, 113606 (2013).

[22] T. Nieddu, V. Gokhroo, and S. Nic Chormaic, Optical nanofibres and neutral atoms, J. Opt. 18, 053001 (2016).

[23] B. Gouraud, D. Maxein, A. Nicolas, O. Morin, and J. Laurat, Demonstration of a Memory for Tightly Guided Light in an Optical Nanofiber, Phys. Rev. Lett. 114, 180503 (2015).

[24] C. Sayrin, C. Clausen, B. Albrecht, P. Schneeweiss, and A. Rauschenbeutel, Storage of fiber-guided light in a nanofiber-trapped ensemble of cold atoms, Optica 2, 353 (2015).

[25] I. H. Deutsch, R. J. C. Spreeuw, S. L. Rolston, and W. D. Phillips, Photonic band gaps in optical lattices, Phys. Rev. A 52, 1394 (1995).

[26] G. Birkl, M. Gatzke, I. H. Deutsch, S. L. Roston, and W. D. Phillips, Bragg Scattering from Atoms in Optical Lattices, Phys. Rev. Lett. 75, 2823 (1995).

[27] M. Weidemüller, A. Hemmerich, A. Görlitz, T. Esslinger, and T. W. Hänsch, Bragg Diffraction in an Atomic Lattice Bound by Light, Phys. Rev. Lett. 75, 4583 (1995).

[28] S. Slama, C. von Cube, B. Deh, A. Ludewig, C. Zimmermann, and P.W. Courteille, Phase-Sensitive Detection of Bragg Scattering at 1D Optical Lattices, Phys. Rev. Lett. 94, 193901 (2005).

[29] A. Schilke, C. Zimmermann, P. W. Courteille, and W. Guerin, Photonic Band Gaps in One-Dimensionally Ordered Cold Atomic Vapors, Phys. Rev. Lett. 106, 223903 (2011). 
[30] F. Le Kien, J. Q. Liang, K. Hakuta, and V. I. Balykin, Field intensity distributions and polarization orientations in a vacuum-clad subwavelength-diameter optical fiber, Opt. Commun. 242, 445 (2004).

[31] F. Le Kien and A. Rauschenbeutel, Anisotropy in scattering of light from an atom into the guided modes of a nanofiber, Phys. Rev. A 90, 023805 (2014).

[32] F. Le Kien and A. Rauschenbeutel, Propagation of nanofiber-guided light through an array of atoms, Phys. Rev. A 90, 063816 (2014).

[33] See Supplemental Material at http://link.aps.org/ supplemental/10.1103/PhysRevLett.117.133603, which includes [34,35], for theoretical simulations of the reflection spectra in presence of broadening or disorder.

[34] C. Lacroûte, K. S. Choi, A. Goban, D. J. Alton, D. Ding, N. P. Stern, and H. J. Kimble, A state-insensitive, compensated nanofiber trap, New J. Phys. 14, 023056 (2012).

[35] F. Le Kien, P. Schneeweiss, and A. Rauschenbeutel, Dynamical polarizability of atoms in arbitrary light fields: general theory and application to cesium, Eur. Phys. J. D 67, 92 (2013).

[36] E. Vetsch, S. T. Dawkins, R. Mitsch, D. Reitz, P. Schneeweiss, and A. Rauschenbeutel, Nanofiber-based optical trapping of cold neutral atoms, IEEE J. Quantum Electron. 18, 1763 (2012).

[37] F. Le Kien, V. I. Balykin, and K. Hakuta, Atom trap and waveguide using a two-color evanescent field around a subwavelength-diameter optical fiber, Phys. Rev. A 70, 063403 (2004).
[38] N. Schlosser, G. Reymond, and P. Grangier, Collisional Blockade in Microscopic Optical Dipole Traps, Phys. Rev. Lett. 89, 023005 (2002).

[39] P. Palittapongarnpim, A. MacRae, and A. I. Lvosvky, A monolithic filter cavity for experiments in quantum optics, Rev. Sci. Instrum. 83, 066101 (2012).

[40] N. W. Ashcroft and N. D. Mermin, Solid State Physics (Saunders, Philadelphia, 1976).

[41] S. Slama, C. von Cube, M. Kohler, C. Zimmermann, and $\mathrm{Ph}$. W. Courteille, Multiple reflections and diffuse scattering in Bragg scattering at optical lattices, Phys. Rev. A 73, 023424 (2006).

[42] A. Görlitz, T. Kinoshita, T. W. Hänsch, and A. Hemmerich, Realization of bichromatic optical superlattices, Phys. Rev. A 64, 011401(R) (2001).

[43] A. Camposeo, M. Anderlini, D. Ciampini, J. H. Müller, D. Wilkowski, E. Arimondo, and H. Ritsch, One-dimensional bichromatic standing-wave cooling of cesium atoms, J. Opt. B 5, S29 (2003).

[44] S. Rist, P. Vignolo, and G. Morigi, Photonic spectrum of bichromatic optical lattices, Phys. Rev. A 79, 053822 (2009).

[45] D. Witthaut and A. S. Sørensen, Photon scattering by a three-level emitter in a one-dimensional waveguide, New J. Phys. 12, 043052 (2010).

[46] H. L. Sørensen et al., Coherent backscattering of light off one-dimensional atomic strings, Phys. Rev. Lett. 117, 133604 (2016). 